\title{
COMMENTARY
}

\section{The elusive task of biomarkers of renal injury}

\author{
Matthieu Legrand ${ }^{* 1,2,3}$, Arnaud Mari,5 and Alexandre Mebazaa',2,3 \\ See related research by Di Somma et al., http://ccforum.com/content/17/1/R29
}

\begin{abstract}
Neutrophil gelatinase-associated lipocalin (NGAL) is one of the most promising candidate biomarkers of renal injury, with expression in renal tissue increasing dramatically after ischemia-reperfusion injury but not in the case of pure pre-renal failure. In a recent issue of Critical Care, Di Somma and colleagues reported that NGAL could improve the classification of acute kidney injury compared with clinical assessment and showed that NGAL was associated with poor prognosis. NGAL may therefore carry different information than biomarkers of renal function. This study finally provides additional evidence for the highly complex relationship between renal function and renal injury.
\end{abstract}

Biomarkers are tools that should aid the physician in diagnosis, in risk stratification with prediction of outcome, and, at best, in clinical decision-making [1]. Because the usual renal biomarkers (for example, serum creatinine and/or urine output) may fail to detect acute kidney injury (AKI) at early onset, there is a current effort to search for and validate new diagnostic biomarkers. Neutrophil gelatinase-associated lipocalin (NGAL) is one of the most promising candidates because its level of expression in renal tissue increases dramatically after ischemia-reperfusion injury but not in the case of pure pre-renal failure [2]. Those data suggest the ability of NGAL to detect renal structural damage. Although first studies have highlighted that NGAL monitoring would perform better than standard biomarkers in detecting or quantifying $\mathrm{AKI}$, other reports have yielded conflicting results and raised concerns regarding the accuracy of NGAL for this purpose [3].

In this context, the study published by Di Somma and colleagues in Critical Care provides important additional

*Correspondence: matthieu.m.legrand@gmail.com

'Burn Center, Department of Anesthesiology and Critical Care, groupe hospitalier universitaire St-Louis-Lariboisière, Assistance Publique-Hôpitaux de Paris, Université Paris 7 Denis Diderot, 1 rue Claude Vellefaux, 75010 Paris, France Full list of author information is available at the end of the article insights [1]. The study included patients admitted to hospital from the emergency room, to assess the diagnosis and prognosis value of plasmatic NGAL using a point-of-care method. The authors attempted a multidimensional approach including the NGAL level combined with the initial clinical assessment of risk of presumed AKI by the caring physician. In a Net Reclassification Improvement analysis, NGAL improved classification of AKI by $32.4 \%$. This improvement occurred mainly by moving patients from the AKI to the no-AKI subgroups. NGAL was confirmed to have a high predictive negative value in this population with low prevalence of AKI.

At first glance, these results suggest that NGAL has an additional diagnostic value to clinical suspicion, which is a crucial requirement for a new biomarker to improve the predictive accuracy of the standard of care. Looking deeper, this study highlights concerns regarding the clinical validation of biomarkers of renal injury. Interestingly, the authors excluded acute renal dysfunctions from AKI, a classification that referred to a subset of disease characterized by transient decline of the glomerular filtration rate with presumed no or minor structural damage (similarly to pre-renal AKI) [4]. This study adds evidence to the recent study from Nejat and colleagues that these classifications based on clinical presumption may be erroneous, with increased serum levels of both biomarkers of renal function and injury in these subsets of patients [5]. Using classifications based on markers of glomerular function (Acute Kidney Injury Network/Risk Injury Failure Loss Endstage/Kidney Disease: Improving Global Outcomes) to validate biomarkers of renal injury introduces the risk of diverting such biomarkers from their true goal and significance, namely to detect organ damage. Using classifications based on a clinical assumption of pure pre-renal mechanism of renal failure obviously further exposes the patient to misclassification of outcome. Although an association exists between organ injury and the inherent loss of function, the relationship is obviously complex and may vary with respect to the causative process $[6,7]$.

The influence of comorbidities adds further complexity. Intriguingly, urine NGAL has been associated with longterm cardiovascular mortality in a cohort of older 


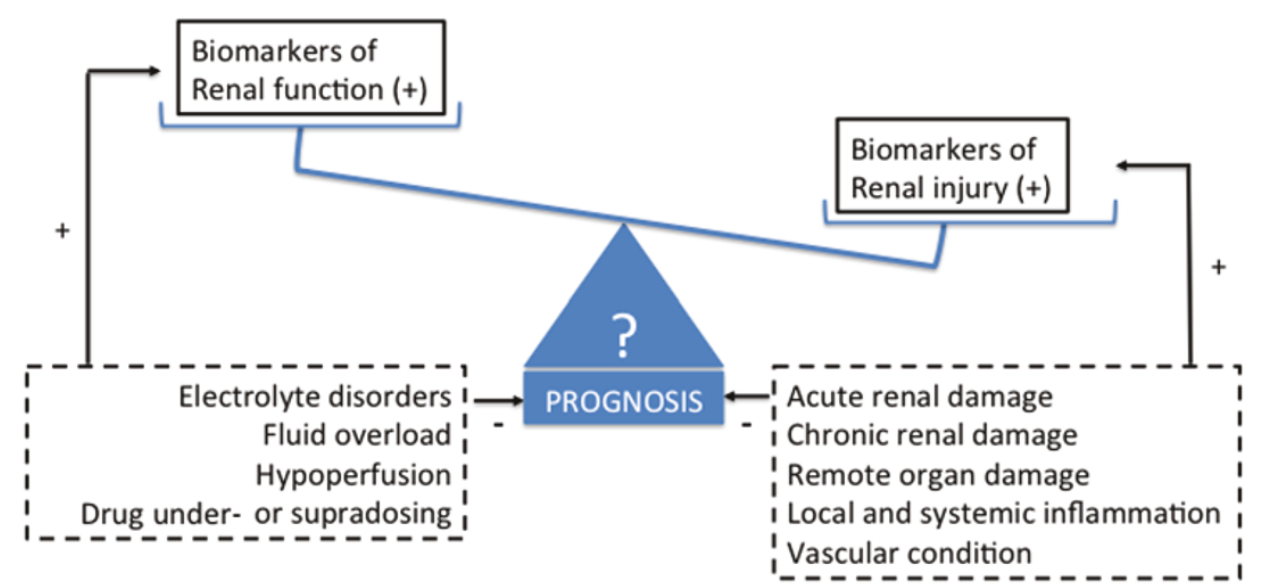

Figure 1. Serum/urine neutrophil-gelatinase associated lipocalin increase might outweigh renal function biomarkers for prediction of adverse outcome. Several lines of evidence suggest that a rise of serum or urine level of neutrophil-gelatinase associated lipocalin (NGAL) might outweigh biomarkers of renal function for prediction of adverse outcome (that is, mortality). Further prospective studies should confirm these findings. Dashed lines enclose potential effectors linking biomarkers of renal function (for example, serum creatinine) and renal injury (for example, NGAL) to poor outcome. These factors can affect the serum level of these biomarkers (+) but can also influence outcome (-). Further research should explore the significance of these associations and will unveil the specificity of the biomarkers toward renal injury.

community-dwelling adults with no past history of clinical cardiovascular disease with a median level of $192 \mathrm{ng} / \mathrm{ml}$, higher than the threshold proposed for detection of AKI in the study by Di Somma and colleagues (that is, $150 \mathrm{ng} / \mathrm{ml}$ ), reflecting a high noise/ signal ratio for the diagnosis of renal failure (which semantically better defines altered glomerular function) [8]. The expectation that a single biomarker (with a much hunted critical threshold) could at the same time capture these mechanisms of disease and could accurately predict the loss of function and/or stage of kidney injury is therefore obviously flawed. By analogy, who would expect increased serum troponin to accurately detect or predict heart failure? Other (bio)markers can do so.

NGAL carries risk information beyond markers of renal function and clinical assessment. In the present study NGAL did not perform better than the estimated glomerular filtration rate or clinical judgment, which share common criteria with the clinical endpoint (namely serum creatinine and estimated glomerular filtration rate) - further suggesting that these biomarkers provide different information, including a prognostic value [9]. Is it time for intensivists to operate the paradigm shift at the bedside in the way we assess AKI by using NGAL to monitor renal damage? Further exploration of the association between biomarkers of renal function, biomarkers of renal injury and prognosis appears a crucial next step before doing so. The results of an ongoing large multicenter study assessing the association between NGAL and 1-year outcome in ICU patients will provide important insights into this issue (FROG-ICU trial, ClinicalTrials.gov:NCT01367093). Exploration of the links between hits, damage and driving forces of renal failure through the sources of different biomarkers [1], identification of the NGAL pathways (that is, systemic inflammation, chronic renal injury, acute renal injury, and so forth) [10] and increased specificity of biomarkers toward renal injury is then required [11,12] (Figure 1). Most importantly, intensivists and emergency physicians should explore whether risk stratification using NGAL will translate in clinical decision-making.

In conclusion, current definitions of AKI are based on renal function biomarkers that carry different information than biomarkers of injury. NGAL can detect renal injury, and does so pretty well. Injury does not always translate into renal failure, however, and the converse holds true. Future research should aim at clarifying what we are really looking at with biomarkers of kidney injury, including NGAL, and what are the clinical implications. Pending these advancements, we certainly have to accept that injury is not function and that in most conditions trying to predict renal failure with biomarkers of injury will remain an elusive task.

\section{Abbreviations}

AKI, acute kidney injury; NGAL, neutrophil gelatinase-associate lipocalin.

\section{Competing interests}

The authors declare that they have no competing interests.

\section{Author details}

'Burn Center, Department of Anesthesiology and Critical Care, groupe hospitalier universitaire St-Louis-Lariboisière, Assistance Publique-Hôpitaux de Paris, Université Paris 7 Denis Diderot, 1 rue Claude Vellefaux, 75010 Paris, France. ${ }^{2}$ Université Paris Diderot, 75475 Paris, France. ${ }^{3} U M R$ 942, Inserm, France. ${ }^{4}$ Département d'Anesthésie-Réanimation, Service de réanimation polyvalente, Hôpitaux de Toulouse, CHU Purpan, 31059 Toulouse, France. ${ }^{5}$ Université Paul Sabatier Toulouse, 3 Fed 4138, EA4564 (MATN), 31062 Toulouse, France. 
Published: 17 April 2013

\section{References}

1. Di Somma S, Magrini L, De Berardinis B, Marino R, Ferri E, Moscatelli P, Ballarino P, Carpenteri G, Noto P, Gliozzo B, Palladino L, Di Stasio E: Additive value of blood neutrophil gelatinase-associated lipocalin to clinical judgement in acute kidney injury diagnosis and mortality prediction in patients hospitalized from the emergency department. Crit Care 2013, 17:R29.

2. Paragas N, Qiu A, Zhang Q, Samstein B, Deng S-X, Schmidt-Ott KM, Viltard M, Yu W, Forster CS, Gong G, Liu Y, Kulkarni R, Mori K, Kalandadze A, Ratner AJ, Devarajan P, Landry DW, D'Agati V, Lin C-S, Barasch J: The Ngal reporter mouse detects the response of the kidney to injury in real time. Nat Med 2011, 17:216-222.

3. Vanmassenhove J, Vanholder R, Nagler E, Van Biesen W: Urinary and serum biomarkers for the diagnosis of acute kidney injury: an in-depth review of the literature. Nephrol Dial Transplant 2013, 28:254-273.

4. Payen D, Legrand M: Can we identify prerenal physiology and does it matter? Contrib Nephrol 2011, 174:22-32.

5. Nejat M, Pickering JW, Devarajan P, Bonventre JV, Edelstein CL, Walker RJ, Endre $\mathrm{ZH}$ : Some biomarkers of acute kidney injury are increased in prerenal acute injury. Kidney Int 2012, 81:1254-1262.

6. Lee S-Y, Lee Y-S, Choi H-M, Ko Y-S, Lee H-Y, Jo S-K, Cho W-Y, Kim H-K: Distinct pathophysiologic mechanisms of septic acute kidney injury: role of immune suppression and renal tubular cell apoptosis in murine model of septic acute kidney injury. Crit Care Med 2012, 40:2997-3006.

7. Takasu O, Gaut JP, Watanabe E, To K, Fagley RE, Sato B, Jarman S, Efimov IR, Janks DL, Srivastava A, Bhayani SB, Drewry A, Swanson PE, Hotchkiss RS:
Mechanisms of cardiac and renal dysfunction in patients dying of sepsis. Am J Respir Crit Care Med 2013, 187:509-517.

8. Daniels LB, Barrett-Connor E, Clopton P, Laughlin GA, Ix JH, Maisel AS: Plasma neutrophil gelatinase-associated lipocalin is independently associated with cardiovascular disease and mortality in community-dwelling older adults: the Rancho Bernardo Study. J Am Coll Cardio/ 2012, 59:1101-1109.

9. Haase M, Devarajan P, Haase-Fielitz A, Bellomo R, Cruz DN, Wagener G, Krawczeski CD, Koyner JL, Murray P, Zappitelli M, Goldstein SL, Makris K, Ronco C, Martensson J, Martling C-R, Venge P, Siew E, Ware LB, Ikizler TA, Mertens PR: The outcome of neutrophil gelatinase-associated lipocalinpositive subclinical acute kidney injury: a multicenter pooled analysis of prospective studies. J Am Coll Cardiol 2011, 57:1752-1761.

10. Bagshaw SM, Bennett M, Haase M, Haase-Fielitz A, Egi M, Morimatsu H, D'amico G, Goldsmith D, Devarajan P, Bellomo R: Plasma and urine neutrophil gelatinase-associated lipocalin in septic versus non-septic acute kidney injury in critical illness. Intensive Care Med 2010, 36:452-461.

11. Legrand M, Collet C, Gayat E, Henao J, Giraudeaux V, Mateo J, Launay J-M, Payen D: Accuracy of urine NGAL commercial assays in critically ill patients. Intensive Care Med 2013, 39:541-542.

12. Mårtensson J, Xu S, Bell M, Martling C-R, Venge P: Immunoassays distinguishing between HNL/NGAL released in urine from kidney epithelial cells and neutrophils. Clin Chim Acta 2012, 9:1661-1667.

doi:10.1186/cc12578

Cite this article as: Legrand $M$, et al:: The elusive task of biomarkers of renal injury. Critical Care 2013, 17:132 